\title{
Design of the tool for periodic not evolvent profiles
}

\author{
Roman Anisimov ${ }^{1, *}$, and Alexander Tarapanov ${ }^{1}$ \\ ${ }^{1}$ Orel State University named after I.S. Turgenev, Komsomolskaya street, 95, Orel, 302026, Russia
}

\begin{abstract}
The new approach to profiling of the tool for processing of parts with periodic not evolvent profiles are considered in the article The discriminatory analysis of periodic profiles including repetition of profile both in the plane of perpendicular axis of part, and in the plane of passing along part of axis is offered. In the basis of the offered profiling method the idea of space shaping by rated surface of product of tool surface lies. The big advantage of the offered approach in profiling is its combination with the analysis of parameters of process of engineering work. It allows to predict the accuracy and surface quality of product with not evolvent periodic profile. While using the offered approach the pinion cutter for processing of wheels with internal triangular teeths and mill for processing of the screw of the counter of consumption of liquid, complex profile of which consists of several formings, have been received
\end{abstract}

\section{Relevance}

In modern mechanical engineering, the products containing complex periodic not evolvent profiles which are widely used to torque transfer in set of mechanisms of machines and devices. Variants of form of profiles vary widely. In addition to traditional evolvent profile (cogwheels with evolvent teeths, connections with the help of involute splines) the triangular, cycloidal, trapezoidal and other profiles are also widely used. (fig. 1) It is caused by constructive purpose of certain mechanism and advantages of each of profiles. For example for cycloidal profile it is the possibility of essential change of contact ratio, reduction of quantity of teeths on gear wheel, without possible cutting, reduction of sliding speed of profiles; for triangular one it is the transfer of big torsion torques at thin-walled plugs and hollow shafts; for spindle gearing it is bigger gear ratio in planetary reducer at rather high efficiency and compactness of transfer. [1,2]

The great variety of helicoids of different profile is applied in products of the machine-building direction. The main advantages of screw mechanisms are the constant gear ratio, the possibility of obtaining large axial forces, the ease of achievement of self-braking, high accuracy of movement, reliability and durability. Helicoids (fig. 1) can also be attributed to periodic profiles on a number of grounds, such as the presence of a complex profile, which is repeated with a certain step, but unlike the gear and spline teeth and joints, it is repeated in the plane passing along the axis of the product. In these products forming may consist of a set of straight lines and curves of the second or more orders of magnitude. The products with a helical surface are also received by the method of running-in which can be both with axis of gravity and without it. When processing helicoids the probability of undercut of profile of product is high that it is necessary to predict and to take into account when designing the tool. The kinematics of processing of helicoids is much more difficult than toothed and slit ones which complicates the process of profiling of the tool and increases the terms of its design. [3] One of the most complex and laborconsuming tasks of tool production is profiling of the tool for processing of periodic not evolvent profiles. The peculiarity of the design of the tool is caused by the lack of unambiguous compliance between the profile of the tool and the profile of the forming surface in any of sections. Even more complex problem is the solution of a number of the related to profiling tasks of optimization of receiving the profile of the tool and machining process. For example, the definition of the admissible errors of the installation of the tool on the machine, the definition of the admissible tool wear. The particularly important this question becomes in the process of designing of the instrument for products with a helical surface. $[4,5,6]$

At the same time the conditions of a rigid competition require reducing the time for project works. Therefore, the fundamental criterion for new profiling methods should be the ability to create computer-aided engineering systems. The existing profiling methods, which differ in degree of formalization and mathematical description, are not always fully amenable to full automation due to inherent contradictions in them. The poor use of computer-aided design tool in domestic engineering affects the quality and cost of the final product seriously. $[4,5,6]$

The optimal solution for automation of project works are interactive software systems in which the possibility of active interaction of the designed system with the person is laid.

\footnotetext{
* Corresponding author: roman.anisim@gmail.com
} 


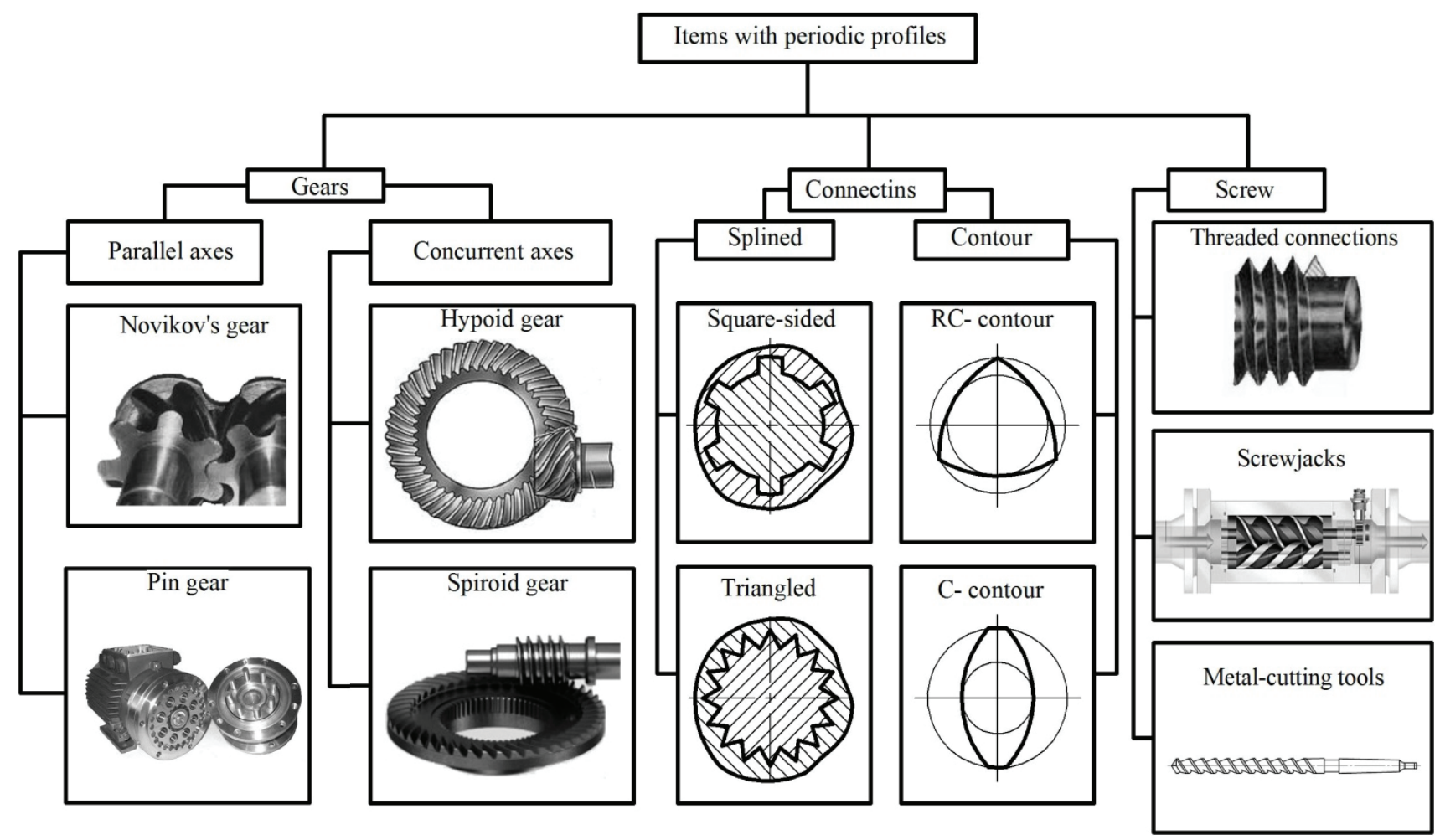

Fig 1. Fragment of the general classification of periodic profiles.

\section{A method of profiling of the tool for periodic not evolvent profiles}

The machining process of complex periodic structures is characterized by a large number of different processing methods and their variants, the complexity of profiling of the cutting edges of the tool, high requirements to surface quality and to kinematic accuracy and errors of a profile even at the preliminary stage of formation.

In the integrated analysis method, the mathematical model of processing by cutting includes the description of the movement of the elementary site of the edge of the cutting tool in the space of formation, the calculation of kinematic changes of face and back angles, the thickness of the cut-off layer, the determination of the components of cutting forces, the wear of the elementary site of the cutting edge, the determination of the errors of machining and the figures of the quality of surface.[7,8]

The diversity to a complex method of the analysis of the shaping of periodic profiles gives that circumstance that each microelement of the studied space has at the same time geometrical and physicomechanical properties of a part and of a tool, possesses functional and space assignment and a certain time span, contains the framework of the model used is the ability to control the shaping in accordance with the specified objectives and constraints. The qualitative indicators of a rated surface, the cutting modes, parameters of the unit of the tool, the time of processing and the cost value can be refered to the latter. [7,8,9]

The method is based on the description of the process of shaping, or processing of a tool by not evolvent periodic surface, where the directing lines of a rated surface act as the cutting edges. For each site of a periodic not evolvent profile there is the thickness of the cut-off layer close to zero at the point N (fig. 2). For each posion of a hollow of a wheel on a certain elementary site of an initial profile of a hollow the point with thickness close to zero will be one. This point can be considered belonging to the treated surface and the tool surface, and its coordinates are adapted coordinates of the tool profile. [8]

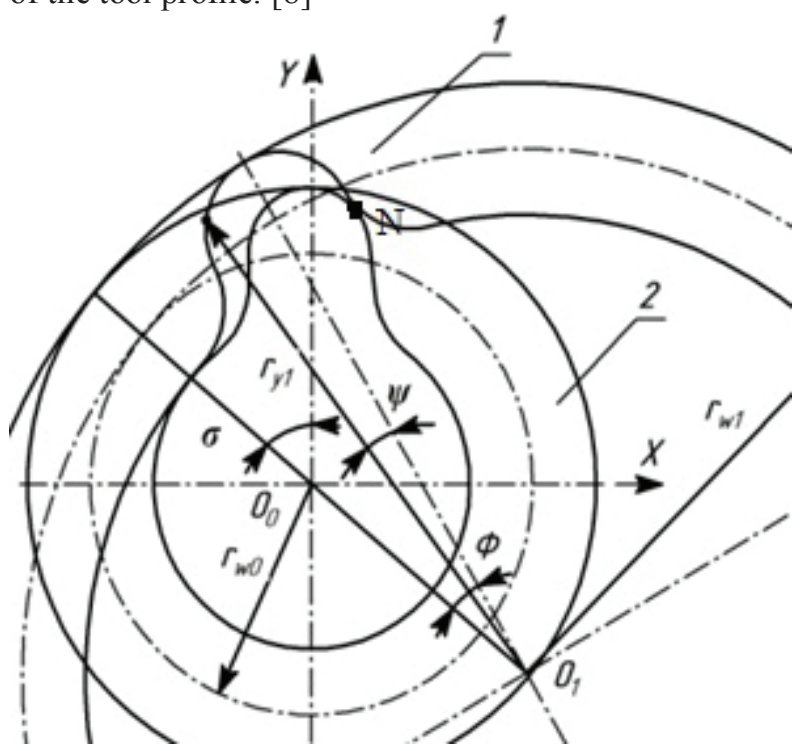

Fig. 2. The scheme of determining of the position of the hollow of the wheel 1 relative to the tooth shaping cutter 2 when profiling.

The schematic of the formation of the generating surface is reduced to the output expression for each 
coordinate that defines the position of each point of not evolvent periodic surface at any moment in time of formation. In this case, all movements are performed by the nominal surface periodic not evolvent profile. This scheme of shaping is constructed by sequential transformation of the original expression that defines the initial profile of the nominal surface. In the process of creating the schema of shaping there is a need to change the direction of the axes or move the center of the coordinates. This is accomplished through the rotation of one of the planes and the movement the whole system along the axes. The transformations used to build a scheme of forming are divided into 2 types. (table 1). $[10,11]$

Table 1. Transformations for creation of the scheme of shaping

\begin{tabular}{|r|l|}
\hline Turn, rotation & $\begin{array}{l}\text { Translational } \\
\text { movement, linear } \\
\text { shift }\end{array}$ \\
\hline$x_{n}=f\left(x_{n-1} ; y_{n-1} ; z_{n-1} ; \psi_{n}\right)$ & $x_{n}=f\left(x_{n-1} ; \psi_{n}\right)$ \\
$y_{n}=f\left(x_{n-1} ; y_{n-1} ; z_{n-1} ; \psi_{n}\right)$ & $y_{n}=f\left(y_{n-1} ; \psi_{n}\right)$ \\
$z_{n}=f\left(x_{n-1} ; y_{n-1} ; z_{n-1} ; \psi_{n}\right)$ & $z_{n}=f\left(z_{n-1} ; \psi_{n}\right)$ \\
\hline
\end{tabular}

where $x_{n-1}, y_{n-1}, z_{n-1}$ - the function equation received from the previous transformation;

$\psi_{n}$ - value of the parameter corresponding to the current transformation.

The index $\mathrm{n}$ denotes the sequence number of the conversion. The direction of rotation in each case is determined by a parameter that can increase or decrease.

The input data of system are: the upper bound and the lower bound of sets $\psi_{1}, \psi_{2}, \ldots, \psi_{n}$ the direction of change (increase or reduction).

Transformations at such operations differ only in the fact that the parameter for creation has the fixed value.

This approach just allows mathematically to provide practically any kinematically difficult scheme, and also to use it for creation of software systems.

Not evolvent periodic profile is set in the edge section. The forming of all profiles applied in mechanical engineering is described by widespread algebraic curves (a straight line, a circle arch, an epicycloid, spirals, an evolvent, etc.) (table 2). The use of such task of forming allows to find any quantity of points of the line of contact of a tool and rated surfaces. $[10,11]$

The profile received by the results of the offered technique is used further for the solution of a direct task - forecasting of parameters of processing by the tool of a rated surface. In the course of the solution of a direct task they receive the values of components of force of cutting which are then used in the basis of obtaining values of accuracy and roughness of the product. In the mathematical apparatus of the solution of a direct task the rigidity of technology system and the deformations arising in it, their influence on accuracy and roughness of the product are considered

Consecutive application of the offered method of profiling of the tool for not evolvent periodic profiles in combination with the mathematical apparatus solving a direct problem allows you to make adjustments to the tool profile taking into account elastic deformations of the technological system. It will lead to the improvement of the quality of the made products - to decrease roughness and to increase accuracy

Table 2. Examples of the algebraic curves in a parametrical form used for creation of the forming line in the edge section of different rated surfaces

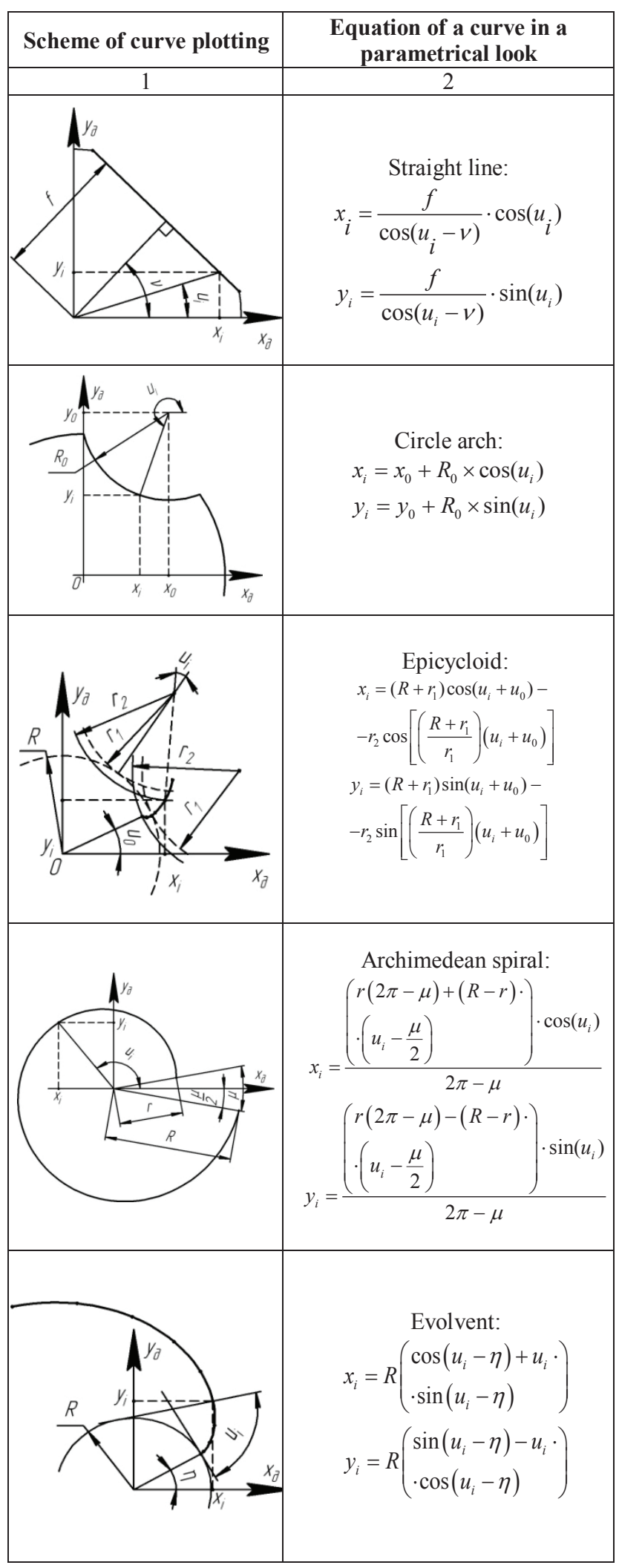




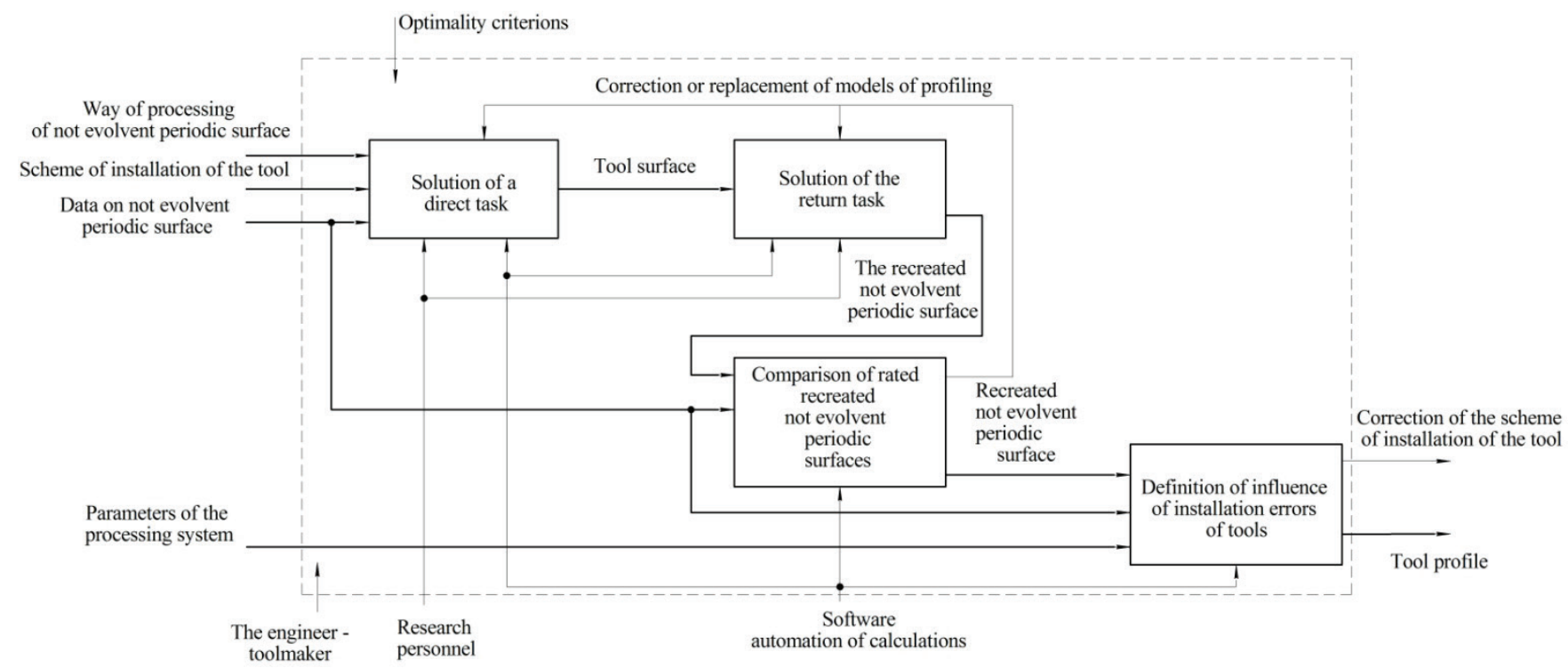

Fig 3. IDEF-0 chart of process of profiling of the tool.

For a more visual demonstration of the stages of profiling with the subsequent forecasting of parameters of the processing of periodic not evolvent profiles the chart of process of profiling of the tool is presented on fig. 3 . The process of profiling of the tool is combined with the analysis of parameters of process of cutting that allows to improve the accuracy and surface quality of the products with periodic not evolvent profiles. [12, 13]

The creation of the profile of the rated tool surface and its correction, definition of the modes of processing, the number of passes, the material of the tool and the model of the machine at which it can be reached, the precision and qualitative characteristics of the processed products set by designers of machinebuilding productions with periodic not evolvent profiles is a consequence of implementation of this chart. This makes it possible to reduce the normal time for the existing technological process, as well as to determine the optimal values of output parameters for the new technological process of processing of the product.

\section{Conclusions}

A new approach to profiling which is based on the spatial process of forming the nominal surface by the tool one with the further analysis of parameters of processing by cutting is offered The profiling method uses the mathematical apparatus adaptable for creating CAD programs. As a result the combination of making and rated surfaces is visually controlled with an accuracy of $10^{-6} \mathrm{~mm}$. It is collectively with the following analysis of processing allows to find the optimal combination of the tool profile, the parameters of the nominal surface of the settings of the tool and parameters of the treatment process.

The proposed approach provides the reduction in financial and time costs of design of the tool and the increase in the accuracy and performance, significantly reducing the implementation of the time of technological processes. In the environment of mathematical and software methods the pinion cutters for a wheel with internal triangular teeths and mills for the liquid consumption counter screw were profiled (fig. 4, fig. 5) [14].

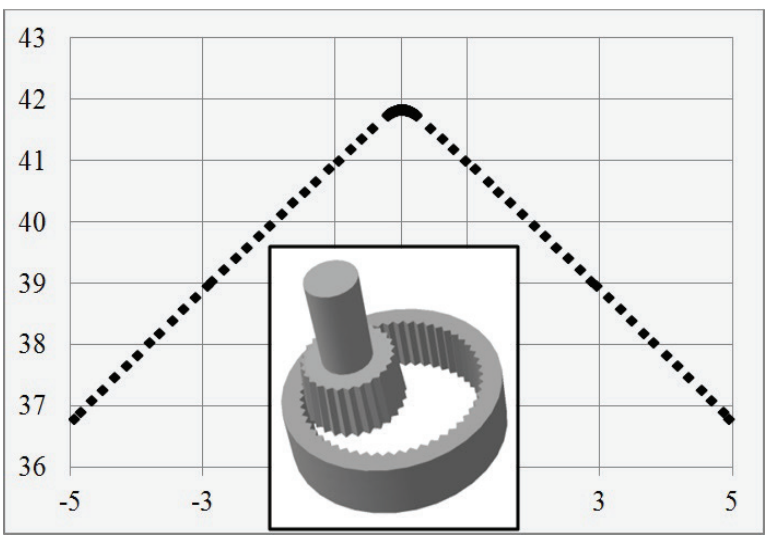

Fig. 4. Results of profiling of the pinion cutter for a triangular profile.

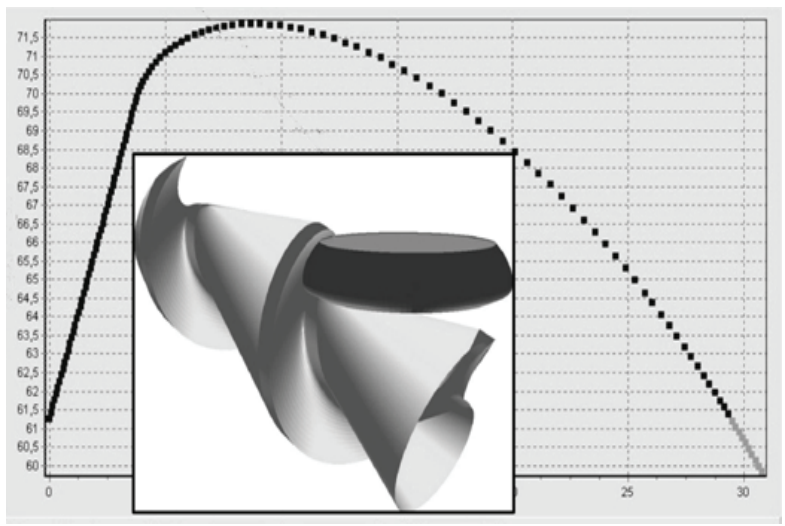

Fig. 5. Results of profiling of the mill for the liquid consumption counter screw. 


\section{References}

1. R.V. Anisimov, A.S. Tarapanov, Modern technologies and the equipment for a gear shaping of wheels with internal teeths, Regional scientific and practical conference of young scientists and graduate students «Scientific capacity of Orlovschina in modernization of an industrial complex of the small cities of Russia», p.4-8 (2010)

2. K D Bouzakis, Manufacturing of cylindrical gears by generating cutting processes, A critical synthesis of analysis methods, CIRP Annals-Manufacturing Technology, 57 p. 676-696 (2008)

3. S.I. Brusov, M.V. Zhuplov, A.S. Tarapanov, The description of an initial helicoid when profiling screw-cutting tools, News Oryol GTU. Fundamental and applied problems of the equipment and technology №3 p., 18-23 (2007)

4. R.V. Anisimov, Improvement of quality of details of vehicles with internal teeths of not evolvent profile, Topical issues of innovative development of a transport complex. Materials of the International scientific and practical conference, 91-95 (2011)

5. G Figliolini, $\mathrm{P}$ Rea, Effects of the design parameters of involute gears generated by rackcutters International Gear Conference 294 (2014)

6. M.V. Zhuplov, Interactive profiling of disk mills for processing of compound half-closed helicoids on the basis of three-dimensional display of the scheme of profiling, News Oryol GTU. Fundamental and applied problems of the equipment and technology № 2-3/274 (560) p.2429 (2009)

7. M.V. Zhuplov, S.I. Brusov, A.S. Tarapanov, An integrated approach in profiling and a research of processing of helicoids, Problems of quality of machines and their competitiveness: materials of the 6th international scientific and technical conference p. 269-270 (2008)

8. R.V. Anisimov, S. I. Mironov, Mathematical display of schemes of cutting and determination of thickness of the cut-off layer when processing not evolvent profiles of cogwheels with internal teeths Fundamental and applied problems of the equipment and technology №2/3(280), p.16-21 (2010)

9. K Nagamura, K Ikejo, F G Tutulan, Design and performance of gear pumps with a non involute tooth profile Proceedings of the Institution of Mechanical Engineers, Part B: J of Engineering Manufacture. 218, 699-711 (2004)

10. R.V. Anisimov, Profiling of gear cutting for wheels with internal not evolvent teeths, Fundamental and application-oriented problems of upgrade of the modern mechanical engineering and metallurgy: The collection of scientific works of the international scientific and technical conference devoted to the 50 anniversary of department of technology of mechanical engineering of LGTU, 11-15 (2012)

11. M.V. Zhuplov, S.I. Brusov, A.S. Tarapanov, The description of an initial helicoid when profiling screw-cutting tools, News Oryol GTU. Fundamental and applied problems of the equipment and technology №3 p. 18-23 (2007)

12. R.V. Anisimov, A.S. Tarapanov, Forecasting of a roughness of processing of cogwheels with internal teeths of not evolvent profile, High technologies in mechanical engineering: Materials of the International scientific and technical conference $\mathrm{p}$. 46-50 (2012)

13. F L Litvin, I Gonzalez-Perez, A Fuentes, K Hayasaka, Design and investigation of gear drives with non-circular gears applied for speed variation and generation of functions, Computer methods in applied mechanics and engineering 45, 3783-3802 (2008)

14. S.I. Brusov, M.V. Zhuplov, A.S. Tarapanov, Option of definition of a profile of a disk mill for processing of a helicoid on the basis of space mathematical display of the scheme of cutting, High technologies in mechanical engineering: theses of reports of the All-Russian scientific and technical Internet conference with the international participation p.135-140 (2008) 